\title{
Cultura escolar em tempos de pandemia e distanciamento social
}

\author{
School culture in times of pandemic and social distancing
}

Cultura escolar en tiempos de pandemia y distanciamiento social

\author{
Maéve Melo dos Santos' \\ https://orcid.org/0000-0003-1867-8I2X \\ Cosme Batista dos Santos² \\ https://orcid.org/0000-0002-9276-5398
}

\begin{abstract}
Resumo: Este artigo trata de um estudo em andamento do grupo de pesquisa "Observatório do ensinoaprendizagem da escrita escolar", da Universidade do Estado da Bahia, que, ao ver a iniciativa das escolas de desenvolverem atividades remotas, sentiu necessidade de investigar como a cultura escolar "sobrevive" nos domicílios e quais dizeres e fazeres simbolizam essa sobrevivência. $O$ objetivo é investigar a cultura escolar em domicílio durante a pandemia da COVID-19 e a percepção de seus atores. A metodologia adotada é a pesquisa quanti-qualitativa, com elaboração de formulários online para estudantes, pais, professores e dupla gestora, e análise do discurso. As conclusões iniciais apontam que os dispositivos de transposição didática da cultura escolar estão ativados mesmo na situação do isolamento. Isso mostra um fortalecimento do papel do educador, da sua capacidade de apreender novos meios de ensino e de superação das condições adversas para fazer-se presente na vida escolar dos estudantes.
\end{abstract}

Palavras-chave: Educação. Pandemia. Cultura escolar.

Abstract: This paper is about an ongoing study conducted by the research group "Observatory of the teachinglearning of school writing", from the State University of Bahia, Brazil, which, when seeing the initiative of schools to develop remote activities, felt the need to investigate how school culture "survives" in the households and what speeches and actions symbolize this survival. The aim is to investigate school culture in the household during the COVID-19 pandemic and the perceptions of its actors. The methodology adopted is a quanti-qualitative research, with the elaboration of online forms for students, parents, teachers and dual managers, and discourse analysis. The initial conclusions point out that the school culture, its didactic transposition devices, are activated even in the situation of social distancing. This shows a strengthening of the role of the educator, his/her ability to learn new means of teaching and to overcome the adverse conditions to make him/herself present in the school life of students.

Keywords: Education. Pandemic. School culture.

'Doutoranda em Crítica Cultural (Pós-crítica) na Universidade do Estado da Bahia (UNEB). Professora Assistente da Universidade Federal do Vale do São Francisco (Univasf). E-mail: maeve.melo@univasf.edu.br.

2Doutor em Linguística Aplicada pela UNICAMP, Professor Pleno da Universidade do Estado da Bahia-UNEB, Campus III, Juazeiro-BA. Emai: cbsantos@uneb.br

Olhar de professor, Ponta Grossa, v. 24, p. I-II, e-16180.05I, 202 I.

Disponível em <https://revistas2.uepg.br/index.php/olhardeprofessor> 
Resumen: Este artículo trata de un estudio en realización del grupo de investigación "Observatorio de la enseñanza-aprendizaje de la escritura escolar" en la Universidad Estatal de Bahía - Brasil que, al ver la iniciativa de las escuelas de desarrollar actividades a distancia, sintió la necesidad de investigar cómo "sobrevive" la cultura escolar en los hogares y qué dichos y actos simbolizan esa supervivencia. El objetivo es investigar la cultura escolar en el hogar durante la pandemia de COVID-19 y las percepciones de los actores. La metodología adoptada es la investigación cuantitativa-cualitativa con la elaboración de formularios en línea para estudiantes, padres, docentes y administradores, y análisis del discurso. Las conclusiones iniciales señalan que la cultura escolar y sus dispositivos de transposición educativa están activados incluso en la situación de aislamiento. Eso muestra un fortalecimiento del papel del educador, de su capacidad de aprehender nuevos medios de enseñanza, y la superación de condiciones adversas para hacerse presente en la vida escolar de los estudiantes.

Palabras-clave: Educación. Pandemia. Cultura escolar.

\section{Introdução}

Certamente, o ano de 2020 marcará, para sempre, a humanidade. Todo o planeta está vivendo, em decorrência da pandemia do novo coronavírus, o isolamento social ou o distanciamento social, como alguns denominam; outros preferem chamar distanciamento físico, pois virtualmente parece que estamos mais próximos uns dos outros. Nunca se fez tantas lives, tantas reuniões online, tantos encontros virtuais, tantas compras no e-commerce, tantos restaurantes delivery. Trata-se de um vírus letal que tem contaminado diariamente pessoas dos mais diversos países ricos, em desenvolvimento ou pobres. No início do surto, a COVID-19 parecia ser um vírus "democrático" do ponto de vista da infecção, atingindo todas as classes sociais, sem distinção de cor, raça, status social, gênero ou sexualidade, porém, com a evolução da pandemia, os dados mostraram outra realidade no que diz respeito ao tratamento e à recuperação. Pesquisas recentes da Associação Brasileira de Saúde Coletiva - ABRASCO (REIS, 2020) revelaram os altos índices de mortalidade entre as comunidades negras, indígenas e pobres, escancarando a grande desigualdade social, fruto de um racismo estrutural no Brasil e no mundo.

Para ter-se uma noção da dimensão da fatalidade desse vírus, em maio de 2020 , existia no mundo cerca de 4 milhões de pessoas infectadas pela COVID-19, nome científico da doença, e quase 300 mil mortes no mundo. O Brasil apresentava 180 mil infectados e cerca de 12.600 óbitos. Até o momento, julho de 2020, já são mais de 14 milhões de pessoas infectadas e mais de 600 mil mortes mundialmente e, no Brasil, considerado o novo epicentro da doença por pesquisadores, já são mais de 2 milhões de infectados e mais de 78.000 óbitos (BBC NEWS, 2020; BRASIL, 2020). De acordo com os dados oficiais de julho de 2020, o Brasil é o segundo país com mais números de óbitos no mundo, em decorrência de uma política nacional negacionista e liberal que tem uma narrativa neoliberal e necropolítica (MBEMBE, 2016), afirmando diariamente que a economia e o Brasil não podem parar.

Diante desse cenário e sem a descoberta da vacina e cura científica da doença, a recomendação geral da Organização Mundial da Saúde (OMS), como medida mais eficaz de combate, é o 
distanciamento social, ou seja, todos ficarem em casa, trabalhando de forma remota. Apenas os serviços essenciais estão liberados, desde que tomadas todas as precauções e medidas de vigilância necessárias. As escolas foram as primeiras a parar em virtude da aglomeração dos alunos em sala de aula. Muitas escolas privadas e sistemas de ensino público têm adotado o ensino remoto por meio de videoaulas, aulas online e outros formatos, como alternativa de manutenção do vínculo afetivo com os estudantes, bem como dar continuidade às atividades escolares. Os estudantes estão em casa sendo convocados a continuar aprendendo em domicílio, sem a presença física do professor e do espaço físico da escola. De acordo com um levantamento da Organização das Nações Unidas para a Educação, a Ciência e a Cultura - UNESCO (2020) sobre a situação da educação no Brasil (por região/estado) durante a pandemia, dos nove Estados do Nordeste, cinco adotaram esse modelo de educação a distância, dois estão planejando e dois não se tem informação a respeito.

Nesse contexto, considerando esse novo cenário pandêmico, surge um problema que merece investigação: Como a cultura escolar "sobrevive" nos domicilios e quais os dizeres e fazeres que simbolizam essa sobrevivência? Este ensaio é parte dos estudos desenvolvidos pelo Observatório do ensinoaprendizagem da escrita, da Universidade do Estado da Bahia (UNEB), do Campus III - Juazeiro, e tem a intenção de analisar a cultura escolar em fase de isolamento social, de forma a refletir sobre o que está sendo construído de possibilidades de ensino durante a pandemia do coronavírus, identificar os dispositivos didáticos empregados para ensinar e aprender e quais as percepções dos atores sociais envolvidos no processo. Para tanto, inicialmente, faremos uma breve discussão em uma perspectiva crítica, analítica e sociointeracionista sobre cultura, cultura escolar e transposição didática, a partir dos estudos de Jean-Claude Forquin. Na sequência, apresentaremos as impressões iniciais dos dados coletados da pesquisa em andamento, realizada por meio de questionário online, sobre o desenvolvimento das atividades remotas em tempos de isolamento social, com $\circ$ intuito de refletir sobre como a cultura escolar resiste e persiste em domicílio. Por estarmos ainda na fase de análise e tratamento dos dados, neste artigo, apresentaremos apenas a visão dos professores.

\section{Cultura e cultura escolar: a perspectiva de Jean-Claude Forquin}

Tomando como base os estudos de Jean-Claude Forquin (2004), École et culture, de 1993, podemos dizer que a cultura escolar está relacionada à perpetuação da cultura da humanidade para que esta não seja esquecida. Para Forquin (2004), a escola é o local por excelência responsável pela gestão e pela transmissão dos conhecimentos acumulados historicamente, porém, ao fazer-se a seleção dos conteúdos a serem estudados na escola, ocorre, inevitavelmente, uma perda significativa do conhecimento acumulado ao longo do tempo. Isso significa dizer que a escola funciona na base do esquecimento, pois sempre alguns conteúdos serão esquecidos, ao não serem selecionados para 
compor o currículo escolar. Essa seleção não se relaciona apenas à herança do passado, mas também à cultura do presente, de forma que algumas atitudes, modos, valores e tipos de conhecimentos presentes são tão relevantes que são escolhidos para compor o currículo escolar. No entanto, Forquin (2004) aponta que, apesar dessa seleção, na escola é feita uma nova seleção, pois cada escola e cada docente faz a seleção dos conteúdos que consideram prioritários. Nesse sentido, o autor pontua a necessidade de reconhecimento da autoridade pedagógica do professor, pois não se pode ensinar de verdade se ele não ensina algo realmente válido a seus próprios olhos. Na perspectiva do estudante, também afirma que nem sempre o que é aprendido pelos alunos corresponde ao que foi ensinado pelos professores, posto que o contexto sociocultural interfere na recepção da mensagem pedagógica ensinada.

Para além da função de transmissora de saberes e da cultura disponíveis em uma sociedade, Forquin (2004) assinala que a cultura escolar também tem o imperativo de realizar a transposição didática dos conhecimentos, de forma que se torna compreensível e assimilável pelos estudantes, posto que a linguagem da ciência não é diretamente comunicável aos alunos, daí que se faz necessária a intervenção e a intermediação da escola por meio dos saberes pedagógicos. Segundo Forquin (1992, p. 32-33): "É preciso a intercessão de dispositivos mediadores, a longa paciência de aprendizagens metódicas e que não deixam de dispensar as muletas do didatismo". Ao citar Michel Verret, ele afirma que: "Toda prática de ensino pressupõe a transformação prévia deste objeto em objeto de ensino" (VERRET, 1975 apud FORQUIN, 1992, p. 33).

Forquim (1992), ao analisar os imperativos didáticos, a cultura escolar e o estudo sobre a divisão do tempo escolar de Verret, considera que as características específicas e próprias da cultura escolar, “[...] a preocupação da progressividade, a importância atribuída a divisão formal (em capítulos, lições, partes e subpartes), a abundância de redundâncias no fluxo informacional [...] às técnicas de condensação (resumos, sínteses documentárias, técnicas mnemônicas)" (FORQUIN, 1992, p. 34), estão impregnadas desse "espírito escolar" que nos propomos a refletir sobre como a escola "sobrevive" nos domicílios e quais os dizeres e fazeres que simbolizam essa sobrevivência. A forma de seleção cultural dos saberes escolares e sua transposição didática, mediada pelos meios tecnológicos e digitais, revelam os desafios da escola atual, em domicílio, tanto para os alunos como para os pais e professores.

Se a universalização do ensino por meio do acesso à Educação Básica a todos os cidadãos brasileiros parecia ser um desafio conquistado ao longo desses últimos 30 anos, principalmente em relação ao Ensino Fundamental, faltando avançar ainda mais no Ensino Médio (PORTELA, 2007) e Educação Infantil (CURY, 2002), a pandemia do coronavírus de 2020 veio nos mostrar que precisamos de uma nova universalização: a universalização do acesso à internet, aos recursos tecnológicos e à 
alfabetização digital. Se estamos vivendo um "novo normal", como tem sido chamado na mídia nos últimos dias, e a cultura escolar está sendo desenvolvida em domicílio, muitas crianças, adolescentes, jovens e adultos estudantes estão fora dessa nova escola, desse "novo normal", pois não têm acesso à internet e não possuem condições de estudar em casa. O Brasil, tão fortemente marcado historicamente pela desigualdade social, buscou assegurar aos estudantes, nesses últimos 30 anos, o acesso à escola física, não a escola virtual. Esse é um novo desafio de um "novo normal" que iremos começar a discutir agora, apresentando as percepções dos professores.

\section{Impressões iniciais e análise dos primeiros resultados}

A pesquisa surgiu de uma indagação no grupo de pesquisa "Observatório do ensinoaprendizagem da escrita escolar", da Universidade do Estado da Bahia (UNEB), Campus III - Juazeiro, que, diante de um cenário de pandemia e distanciamento social, vendo a iniciativa das escolas do Vale do São Francisco, mais especificamente da Rede Integrada de Desenvolvimento (RIDE PetrolinaJuazeiro) ${ }^{3}$, de desenvolverem atividades remotas durante esse período, sentiu a necessidade de buscar respostas para um problema que merece investigação: Como a escola "sobrevive" nos domicílios e quais os dizeres e fazeres que simbolizam essa sobrevivência? Para tanto, elaboramos formulários específicos para estudantes, pais, professores e dupla gestora (diretores e coordenadores), com questões abertas e fechadas, que foram disponibilizados por meio das redes sociais, solicitando que respondessem ao questionário de forma online. O questionário foi dividido em duas partes: a primeira traz perguntas relacionadas à cultura escolar em domicílio e a segunda sobre as práticas de letramento no período de distanciamento social. Para efeito deste artigo, iremos analisar apenas a primeira parte das respostas dos professores, ficando para um próximo texto as percepções dos estudantes, pais e dupla gestora (diretores e coordenadores).

Os professores e as professoras que responderam ao questionário online são dos Estados da Bahia, de Pernambuco, em maior parte, do Ceará, de Piauí e de Minas Gerais, tanto da rede pública como privada. Dos 71 docentes que responderam à pesquisa virtualmente, $42 \%$ moram na cidade de Petrolina (Pernambuco), 2I,I3\% em Juazeiro da Bahia, 8,45\% na cidade de Ocara (Ceará), e o restante (28, $17 \%)$ em diversas cidades dos Estados já citados. O fato de a localização do Campus III-UNEB ser na cidade de Juazeiro-BA, vizinha do município de Petrolina-PE, justifica a predominância dessas duas cidades. Cabe destacarmos a dificuldade com esse tipo de pesquisa online, relacionada ao retorno dos

\footnotetext{
${ }^{3}$ A RIDE Petrolina-Juazeiro é composta pelos municípios de Lagoa Grande, Orocó, Petrolina, Santa Maria da Boa vista, no Estado de Pernambuco, e pelos municípios de Casa Nova, Curaça, Juazeiro e Sobradinho, no Estado da Bahia. Disponível em: http://www.planalto.gov.br/ccivil_03/_ato2019-2022/2020/decreto/DI0296.htm. Acesso em: 10 jul. 2020.
}

Olhar de professor, Ponta Grossa, v. 24, p. I-II, e-16180.05I, 2021.

Disponível em <https://revistas2.uepg.br/index.php/olhardeprofessor> 
respondentes, tendo em vista o acúmulo de atribuições dos docentes durante o período da pandemia e a chuva torrencial de informações que chegam a cada minuto nos e-mails, nas redes sociais e no WhatsApp. Outro fator que merece destaque é que o fato de que, sendo o questionário online e tendo sido disponibilizado nas redes sociais, permitiu a participação de professores para além da área de atuação do grupo de pesquisa.

Aproximadamente $70 \%$ dos professores e das professoras respondentes trabalham no Ensino Fundamental (anos iniciais e finais) e Ensino Médio, e 30\% trabalham nas demais modalidades de ensino: Educação Infantil, Ensino Superior, Educação de Jovens e Adultos ou Educação Inclusiva. Destes 30\%, 18\% atuam em diversas modalidades, paralelamente. Cerca de $65 \%$ estão entre a faixa etária de 25 e 45 anos de idade, o que equivaleria ao início e meio da carreira docente, e $27 \%$ estão entre 45 e 55 anos, mais próximos da aposentadoria. Quanto à formação acadêmica, mais de 50\% possuem especialização e cerca de $20 \%$ já têm Mestrado ou Doutorado, o que significa que estamos vivendo um outro momento formativo no país, talvez fruto dos investimentos nas últimas décadas (GATTI; BARRETTO; ANDRÉ, 20I I) em formação docente. Apenas a título de memória, o desafio quando da aprovação da Lei de Diretrizes e Bases da Educação, Lei № 9.394, de 20 de dezembro de 1996, era assegurar que as professoras da Educação Infantil e do Ensino Fundamental (anos iniciais) cursassem a Licenciatura em Pedagogia (BRASIL, 1996). Da mesma forma, quando questionados sobre "Há quantos anos exerce a profissão docente", observamos que $66,38 \%$ têm entre I e I5 anos de experiência, I2,68\% possuem entre 16 e 20 anos de carreira e $21,12 \%$ têm mais de 21 anos de experiência, reforçando a compreensão de que a maior parte dos respondentes é formada por professores e professoras que estão no início ou no meio de sua carreira.

Todos os participantes estão vivenciando a suspensão das aulas durante a pandemia. Essa situação tem trazido novas demandas para os docentes; no entanto, dos 7 I respondentes, 2 I (29,58\%), dos quais a maioria atua na cidade de Petrolina-PE, apontaram que não estão tendo novas demandas, pois as escolas não estão desenvolvendo aulas remotas. O restante está com novas demandas relacionadas à produção de aulas online, de videoaulas, de vídeo conferências, de uso das novas ferramentas tecnológicas e de redes sociais, dentre outras. Todavia, como os professores e as professoras se sentem em relação a essas novas demandas? Antes de responder a esse questionamento, informamos que, de acordo com os dados da pesquisa, mais de $50 \%$ são responsáveis pela elaboração, produção e acompanhamento das atividades remotas junto aos estudantes, mas apenas 15,5\% informaram que "Existe uma equipe específica para a elaboração das atividades, eu acompanho a resolução das atividades pelos alunos e corrijo". Essas atividades são enviadas aos estudantes pelo WhatsApp, cerca de 50\%; por meio de plataforma de ensino, aproximadamente 33\%; pelo celular, em torno de $15 \%$; e por e-mail, próximo de 10\%.

Olhar de professor, Ponta Grossa, v. 24, p. I-II, e-16180.05I, 2021.

Disponível em <https://revistas2.uepg.br/index.php/olhardeprofessor> 
Algumas perguntas do questionário dos professores se referiam ao desenvolvimento das atividades pelos estudantes. Sobre isso, destacamos alguns pontos relevantes: a) $62 \%$ dos estudantes estão realizando as atividades pelo celular; $23,9 \%$, pelo notebook; e 12,7\%, no computador de mesa; b) $40 \%$ dos estudantes estão sendo auxiliados pelos pais ou responsáveis quanto à orientação das atividades escolares; e $26,8 \%$ estudam sozinhos, seguindo as orientações das videoaulas, plataformas de ensino e outros.

Quanto aos processos de ensino e de aprendizagem, as formas de acompanhamento e de avaliação, os dados apresentados convidam-nos a refletir sobre o tipo de aprendizagem que está sendo desenvolvida em domicílios e os desafios impostos tanto para os professores como para os estudantes, pais e responsáveis. De modo geral, os professores estão avaliando por meio da correção das atividades enviadas (22,53\%), ou por meio de vídeo conferências, conversas em grupos do WhatsApp, envio de fotos ou vídeos realizados pelos alunos (19,71\%). Mais de 30\% afirmaram que não estão conseguindo avaliar ou que isso ocorre com dificuldades diversas. Outros professores afirmaram que não estão tendo avaliações (22,53\%), e alguns disseram apenas "bom", "razoável” ou respostas confusas (12,66\%).

Essa dificuldade em avaliar a distância é perceptível também ao analisarmos as respostas da pergunta seguinte: "Você considera que os estudantes estão conseguindo aprender os conteúdos escolares em domicílio durante esse período de isolamento social?". Parte dos professores considera que os alunos não estão aprendendo (34\%), outra parte considera que sim (32\%), outros apenas parcialmente (I5\%) e o restante ou não estão dando aulas (I3\%) ou não conseguem avaliar (6\%). Ainda sobre a aprendizagem, quando questionados(as) sobre quais as dificuldades que os seus estudantes estão encontrando nesse período de isolamento para aprender os conteúdos do componente curricular, mais de $50 \% \operatorname{dos}(\mathrm{as})$ professores e professoras responderam: a) falta de recursos necessários para o devido acompanhamento dos conteúdos, por exemplo: computador, internet de qualidade, celular, dentre outros; b) o ensino a distância, pois a maioria dos alunos necessita de explicações e acompanhamento mais de perto; c) falta de interação com o professor para tirar dúvidas, levando a uma dificuldade que é afetiva, presencial, metodológica; d) analfabetismo digital. Alguns docentes, 23,95\%, afirmaram "não sei, não está tendo aulas ou nenhuma", e 14,08\% disseram que os alunos tinham dificuldades diversas, a exemplo de "muitas atividades, explanação dos conteúdos, falta de rotina", dentre outras.

Os dados iniciais apontam ainda que a maioria dos docentes se sente "preparado(a) para propor e acompanhar as atividades escolares que serão realizadas em casa pelos estudantes durante a pandemia" (53,5\% responderam "sim"; 35,2\% responderam "não"; 8,45\% "parcialmente”, e 2,82\% afirmaram que “ainda não realizei nenhuma atividade remota"). A priori, é possível fazer uma leitura de que os(as) docentes, mesmo não tendo domínio das novas tecnologias, são professores(as) qualificados(as) e se 
sentem preparados(as) para acompanhar essa nova demanda. Apesar de mais de $50 \%$ se sentirem preparados, ao analisarmos as respostas do questionamento "Esse período de pandemia tem gerado alguma angústia em você quanto ao futuro da profissão docente?", 63,38\% afirmaram que estão sentindo angústia e 35,21\% afirmaram que não.

Avançando na análise seguinte "Como você imagina que será o papel do professor após a pandemia?", algumas respostas reforçam esses sentimentos presentes nos discursos transcritos anteriormente: a) 25,35\% apontaram que "será dificil, complicado, trabalhoso, exaustivo, árduo, pesado, sobrecarregado, com muitas cobranças"; b) 22,54\% disseram que terão "novas ferramentas e novas possibilidades; práticas didáticas estendendo-as para outros ciberespaços; aulas mais interativas; dinâmicas, teremos outras metodologias"; c) 18,31\% afirmaram que o "professor do pós-pandemia precisa ser mais humanizado, provocador, propositor, acolhedor, construtor coletivo, socialmente engajado e não um 'entregador de atividades" d) 15,49\% declararam: "O mesmo de antes da pandemia"; e) o restante varia entre não sei responder ou importante, fundamental e será mais valorizado pela sociedade.

Se, por um lado, eles e elas se sentem angustiados e vivem as consequências do cansaço de uma Sociedade do Desempenho (HAN, 20I5), por outro, a pesquisa revela que ainda conseguem ter uma percepção positiva da sua profissão docente. Quase $80 \%$ dos professores e das professoras consideraram que o "isolamento social provocado pelo contexto da pandemia mudou a forma como as pessoas percebem a profissão docente", afirmando, dentre várias respostas, que, durante esse tempo em que os pais estão passando com os filhos para ensinar as atividades escolares, muitos estão percebendo a relevância do papel do professor e que a tecnologia pela tecnologia não ocupa o lugar docente. Outros não consideram que vai haver muitas mudanças quanto à percepção do papel do professor. $\mathrm{Da}$ mesma forma que enxergam que a pandemia mudará a forma como a sociedade percebe a profissão docente, $80,28 \%$ dos professores e das professoras também passaram a ver sua profissão com novos olhos, novos horizontes. Uns sinalizaram que sentiram falta da presença dos alunos, "quanto faz falta a sala de aula, os alunos", sentiram a necessidade de olhar no olho do aluno". Outros se perceberam aprendentes e afirmaram: "Me sinto mediadora e aprendiz ao mesmo tempo". Contudo, houve aqueles docentes que afirmaram ser o sentido de humanidade que prevalecerá na educação futura póspandemia.

Os dados iniciais da pesquisa mostram, também, que cerca de $40 \%$ dos entrevistados pensam que a cultura escolar não irá mudar, continuará da mesma forma, não sabem nem responder ou irá piorar em virtude das cobranças externas de cumprimento dos prazos e dos conteúdos. Em contraponto, quase $60 \%$ dos docentes têm visão positiva da cultura escolar pós-pandemia, seja no campo dos avanços tecnológicos e digitais nas escolas, seja na valorização docente pelos pais e pela sociedade de modo geral, ou, ainda, em uma percepção mais humana, solidária e harmoniosa no 
ambiente escolar ou mesmo pelos aspectos relacionados aos procedimentos de segurança e vigilância sanitária nesses espaços. Esse sentimento resulta de uma percepção de que o "novo normal" precisa vir repleto de humanidade.

\section{Considerações finais}

Este estudo, do grupo de pesquisa "Observatório do ensino-aprendizagem da escrita", realizado neste momento de pandemia, visou mostrar o esforço dos educadores e das educadoras para ensinar e fazer aprender os conteúdos escolares em domicílio. As respostas que coletamos por meio dos questionários online apontaram que os professores e as professoras e estudantes estão conseguindo manter uma cultura escolar no ensino a distância e que a cultura escolar no contexto da pandemia recorre a vários dispositivos de transposição didática, incluindo o livro didático, as tecnologias da comunicação e da informação e outros tantos.

A cultura escolar, seus dispositivos, estão ativados mesmo na situação do isolamento. Isso mostra, a rigor, um fortalecimento do papel do educador e da educadora, da sua capacidade de apreender novos meios de ensino e de superação das condições adversas para fazer-se presente na vida escolar dos estudantes. Defendemos a "autoridade pedagógica do professor" para garantir o ensino-aprendizagem do estudante, seja antes, durante ou pós-pandemia, passando desde a seleção dos conteúdos que serão estudados à transposição didática desses saberes para os novos contextos onde ocorrerão as aprendizagens, nesse caso, os domicílios. Além disso, há a necessidade de adaptação dos saberes a ensinar aos estados e às formas dos conhecimentos prévios e à idade dos aprendizes, pois implica saber fazer o aluno aprender o conteúdo, no contexto da pandemia, por meio dos meios tecnológicos e digitais.

As impressões iniciais da pesquisa, a partir do olhar docente, apontam alguns caminhos possíveis, descritos pelos próprios atores: o da renovação, o da superação, de oportunizar uma cultura escolar pós-pandemia "mais vasta e complexa...isso é bom! Todos precisam se readaptar ao novo. Uma nova construção". Precisamos reinventar-nos e essa reinvenção precisa estar permeada de humanidade.

Os dados coletados dos professores apontam resultados bastante curiosos a respeito dos desafios da escola no pós-pandemia. Se, por um lado, é inevitável os ganhos de inovação e de aprimoramento do ensino mediado pelos meios tecnológicos; por outro, é também inevitável que o retorno seja também uma retomada da competitividade, no Brasil, em especial, da concorrência por resultados com médias aceitáveis nas avaliações em rede e por financiamentos. A realidade de antes, então, parece retornar com a força de apagar os ganhos de aprimoramento da cultura escolar em sua relação com a família no percurso do isolamento social.

De qualquer forma, os resultados futuros, embora seja uma expectativa de grande parte dos 
docentes na pesquisa, serão revistos após o retorno. O Observatório do ensino-aprendizagem da escrita escolar terá, sem dúvida, um trabalho imenso para evidenciar os significados da cultura escolar em diferentes cenários e, também, extrair respostas para ajudar o poder público a tirar proveito das experiências inovadoras ou não, porém todas comprometidas com o sucesso escolar dos estudantes de diferentes realidades sociais e econômicas.

\section{Referências}

BBC NEWS. Coronavírus: o mapa que mostra o alcance mundial da doença. 2020. Disponível em: https://www.bbc.com/portuguese/internacional-5 I7/8755. Acesso em: I5 jul. 2020.

BRASIL. Covid-I 9 no Brasil. 2020. Disponível em: https://susanalitico.saude.gov.br/extensions/covid19_html/covid-19_html.html. Acesso em: I5 jul. 2020.

BRASIL. Decreto n. 10.296, de 30 de março de 2020. Dispõe sobre a Região Administrativa Integrada de Desenvolvimento do Polo Petrolina e Juazeiro e institui o seu Conselho Administrativo. Diário Oficial da União. Disponível em: http://www.planalto.gov.br/ccivil_03/_ato20192022/2020/decreto/D I0296.htm. Acesso em: I 0 jul. 2020.

BRASIL. Lei $\mathbf{N}^{\circ}$ 9.394, de 20 de dezembro de 1996. Estabelece as diretrizes e bases da educação nacional. Brasília: Presidência da República, Casa Civil, Subchefia para Assuntos Jurídicos, 1996. Disponível em: http://www.planalto.gov.br/ccivil_03/leis/19394.htm. Acesso em: 20 jul. 2020.

CURY, C. R. J. A educação básica no Brasil. Educação \& Sociedade, Campinas, v. 23, n. 80, p. 168 200, set. 2002. Disponível em: https://www.scielo.br/pdf/es/v23n80//2929.pdf/. Acesso em: 20 jul. 2020.

FORQUIN, J. C. École et culture. EPS et Société Infos, n. 26, oct. 2004. Disponível em: http://www.epsetsociete.fr/IMG/pdf/forquin_I_.pdf. Acesso em: 20 jul. 2020

FORQUIN, J. C. Saberes escolares, imperativos didáticos e dinâmicas sociais. Teoria e Educação, Porto Alegre, n. 5, p. 28-49, 1992.

GATTI, B.; BARReTTO, E. S. S.; ANDRÉ, M. Políticas docentes no Brasil: um estado da arte. Brasilia: UNESCO, $201 \mathrm{I}$.

HAN, B. C. Sociedade do cansaço. Petrópolis: Vozes, 2015.

MBEMBE, A. Necropolítica. Arte \& ensaios, Rio de Janeiro, n. 32, p. I23-I5I, set. 20I6. Disponível em: https://revistas.ufrj.br/index.php/ae/article/view/8993/7/69. Acesso em: 20 jul. 2020.

ORGANIZAÇÃO DAS NAÇÕES UNIDAS PARA A EDUCAÇÃO, A CIÊNCIA E A CULTURA. Situação da educação no Brasil (por região/estado). 2020. Disponível em: https://pt.unesco.org/fieldoffice/brasilia/covid- I 9-education-Brasil. Acesso em: 20 jul. 2020.

PORTELA, R. Da universalização do ensino fundamental ao desafio da qualidade: uma análise histórica. Educação \& Sociedade, Campinas, v. 28, n. 10, p. 661-690, out. 2007. DOI: https://doi.org/I0.1590/S0I0I-73302007000300003 
REIS, T. Cenário brasileiro da equidade em saúde em meio à Covid-19 compõem panorama internacional em publicação. 2020. Disponível em: https://www.abrasco.org.br/site/noticias/cenario-brasileiro-da-equidade-em-saude-em-meio-a-covid19-compoem-panorama-internacional-em-publicacao/49773/. Acesso em: I5 jul. 2020.

Recebido em: 16 de junho de 2020.

Versão corrigida recebida em: 0I de dezembro de 2020.

Aceito em: 0I de dezembro de 2020.

Publicado online em: 04 de junho de 202I.

(c) (i) 